\title{
The Early Start of Gonadotropin-releasing Hormone Antagonist Versus Flexible Antagonist Ovarian Stimulation Protocol in Poor Responders: A Randomized Clinical Trial
}

\section{Tayebeh Esfidani}

Tehran University of Medical Sciences

\section{Ashraf Moini}

Royan Institute

Arezoo Arabipoor

Royan Institute

Maryam Farid Mojtahedi

Tehran University of Medical Sciences

\section{Shima Mohiti}

Royan Institute

Ladan Kashani ( $\sim$ kashani_ladan@tums.ar.ir)

Tehran University of Medical Sciences

\section{Research}

Keywords: conventional antagonists, poor responders, IVF/ICSI outcome, early follicular

Posted Date: August 6th, 2020

DOI: https://doi.org/10.21203/rs.3.rs-53355/v1

License: (c) (i) This work is licensed under a Creative Commons Attribution 4.0 International License.

Read Full License 


\section{Abstract}

Background: Determining the initiation day of antagonist administration is an important and challenging issue and different results have been reported in the previous studies. The present study was designed to compare the controlled ovarian hyperstimulation $(\mathrm{COH})$ cycles outcomes of the early start of gonadotropin-releasing hormone antagonist (GnRH-ant) protocol with conventional flexible $\mathrm{GnRH}$-ant protocol in patients with poor ovarian response (POR) diagnosis.

Methods: This randomized controlled trial was performed on infertile women who were diagnosed as poor ovarian responder in IVF/ICSI cycles at Arash Women's Hospital affiliated to Tehran University of Medical Sciences between September 2019 and May 2020. POR was defined according to the Bologna criteria and the eligible women were randomly allocated into an experimental (early onset GnRH-ant) and control (flexible GnRH-ant) groups. The women in the experimental group received recombinant gonadotropins (150-225 IU) and GnRH-ant $(0.25 \mathrm{mg})$ simultaneously on the second day of the cycle. In control group, the starting and dose of gonadotropins were similar but daily administration of $\mathrm{GnRH}$-ant was initiated when the leading follicle diameter was $\geq 13 \mathrm{~mm}$. The IVF/ICSI outcomes were compared between groups.

Results: Totally, 116 women were randomly assigned to early start antagonist group $(n=58)$ and control group $(n=58)$. The analysis showed that the two groups did not have a statistically significant difference in terms of the ovarian stimulation duration and the total dose of used gonadotropins. Although, the total number of MIl oocytes in early start of antagonist group was significantly higher than that of in control group $(P=0.04)$. In the following, clinical and ongoing pregnancy rates per $E T$ in early start of antagonist group were significantly higher than those of in control group ( $P=0.02$ and $P=0.03$, respectively); however, the implantation and miscarriage rates were similar between group.

Conclusion: The early onset of GnRH-ant protocol can improve the number of retrieved and MIl oocytes rates and probably the pregnancy outcome after fresh embryo transfer in POR patients; however, larger randomized clinical trials are required to compare the pregnancy outcomes after this approach with other $\mathrm{COH}$ protocols with considering cost-effectiveness issue.

Trial registration: IRCT, IRCT20110731007165N9. Registered 8 February 2020 - Registered while recruiting, https://www.irct.ir/trial/45024.

\section{Background}

Despite a large number of studies regarding poor ovarian response (POR) in assisted reproduction (ART) cycles in the last 20 years, there is still debate to determine the best and most effective protocol for controlled ovarian stimulation (COS) for these patients. Among the various methods of COS recently, the administration of gonadotropin-releasing hormone ( $\mathrm{GnRH}$-ant) regimen in poor responders had 
numerous benefits with decreased stimulation duration and total amount of gonadotrophins required, without symptoms of hormonal withdrawal and no ovarian cyst formation (1-3). The main advantage of applying the $\mathrm{GnRH}$-ant in the treatment of poor responder is that it prevents premature surge of luteinizing hormone( $\mathrm{LH})$ without decreasing the suppressive action on the pituitary gland as that observed with treatment by $\mathrm{GnRH}$ agonist $(2,4)$.

On the basis of the initiation day of administration, the $\mathrm{GnRH}$-ant protocol could be divided into early onset (before day 6 of stimulation) or late onset (after day 6 of stimulation) $(5,6)$. Early follicular starting of $\mathrm{GnRH}$-ant had been reported to decrease exposure to $\mathrm{LH}$ and estradiol during controlled ovarian stimulation which is negatively affecting the chance of pregnancy (4). Moreover, there is inhibition of increment of interphase peak of follicle-stimulating hormone (FSH) by administration of the antagonist at starting of the menstrual cycle leading to better synchronization of growing follicles cohort and consequently increasing the yield of oocytes $(4,7,8)$.

Determining the initiation day of antagonist administration is an important and challenging issue and different results have been reported in the previous studies (5, 9-11). Inal and colleagues (2017) in a flexible antagonist protocol showed that early initiation of antagonists was more cost-effective for number of used gonadotropins and the number of stimulation days (5).

Considering the limited number of clinical trial studies in this field, the present study was designed as randomized clinical trial to compare the effects of the early onset $\mathrm{GnRH}$ antagonist protocol with flexible $\mathrm{GnRH}$ antagonist protocol on vitro fertilization/ intra-cytoplasmic sperm injection (IVF/ICSI) cycles outcome in patients with POR diagnosis.

\section{Materials And Methods}

This randomized controlled trial was performed on infertile women with poor ovarian response (POR) diagnosis who underwent IVF/ICSI cycles at Arash Women's Hospital affiliated to Tehran University of Medical Sciences between September 2019 and May 2020. The trial protocol was approved by the Review Boards and Ethics Committee of Tehran University of Medical Sciences (ethics reference number: IR.TUMS.MEDICINE.REC.1398.544) and was registered in Iranian Registry of Clinical Trials Website (www.irct.ir, IRCT20110731007165N9).

The women with POR diagnosis undergoing IVF/ICSI cycles were determined on the basis of Bologna criteria (12) and existence of at least two of the following criteria: 1) a previous history of POR (retrieved oocytes $\leq 3$ ) in a conventional stimulation protocol, 2 ) advanced maternal age ( $\geq 40$ years) or any other risk factors for POR (e.g. a history of ovarian surgery) and 3) abnormal ovarian reserve test (i.e. antral follicle count (AFC) $<5$ follicles or anti-Müllerian hormone $(\mathrm{AMH})<1.1 \mathrm{ng} / \mathrm{ml}$ ). The patients with age over 44 years, uterine factor and/or severe male factor infertility, hypothalamic amenorrhea, chronic diseases, preimplantation genetic detection diagnosis, history of recurrent miscarriage and repeated implantation failure were excluded from study. The eligible patients were randomly allocated into two groups: early antagonist protocol (intervention group) and flexible antagonist protocol (control group). The permuted 
block randomization was conducted by the statistician advisor with a computer-generated list. The type of treatment was placed in sealed envelopes and the assignment to intervention and control groups were performed by the out-of-study nurse. Each patient was participated in the study only once and if she had signed the written informed consent.

The pituitary down-regulation was achieved and maintained by the $\mathrm{GnRH}$-ant protocol and controlled ovarian hyperstimulation ( $\mathrm{COH}$ ) was performed using the recombinant follicle stimulating hormone FSH ( $\mathrm{rFSH}$ ) (Gonal-F, Merck-Serono) and human menopausal gonadotropin (hMG; Menopur; Ferring) for all of patients. In both study groups serial two-dimensional follicle monitoring by transvaginal ultrasonography (Philips Affiniti 70 machine with a C10-3v Pure-Wave endovaginal probe) and hormonal assay (as needed) were performed.

The women in early antagonist group received rFSH (150-225 IU) and GnRH-ant (0.25 mg) (Cetrotide : Merck-Serono, or Orgalutran, MSD) simultaneously on the second day of the cycle. The patients in control group received daily injections of recombinant FSH (150-225 IU) from day 2 of the cycle and the GnRHant (Cetrotide: $0.25 \mathrm{mg}$ daily) was initiated when the leading follicle diameter was $\geq 13 \mathrm{~mm}$. In both groups, when at least two dominant follicles with $17 \mathrm{~mm}$ or greater in diameter was observed in ultrasound monitoring, the final oocyte maturation was triggered by human chorionic gonadotropin (hCG) (10000 IU, Choriomon, IBSA). The serum estradiol, progesterone and LH levels were measured at two points: baseline assessment (day 1 or 2 of menstrual cycle) and hCG administration day. The all-freeze strategy was applied for patients with progesterone level $\geq 1.2 \mathrm{ng} / \mathrm{ml}$ on hCG administration day.

The ovarian puncture was carried out 34-36 hours after hCG injection and IVF/ICSI process was then applied in accordance with our standards clinical procedures. The embryos quality were evaluated on the basis of Cummins et al.'s (13) criteria with detecting the number of blastomeres, fragmentation, multinucleation and symmetry on the third day after oocyte retrieval. On the basis of the women's age and embryos quality, up to three embryos were transferred at cleavage stage (day 3 after ovum pickup). Luteal phase was supported by using vaginal progesterone suppositories $400 \mathrm{mg}$ BID (Cyclogest ${ }^{\circledR}$, Actoverco, Iran) daily for 14 days until to pregnancy test day. In case of the positive pregnancy, vaginal progesterone was administrated until 10 weeks of gestation.

The primary outcome was the total number of retrieved and metaphase II (MII) oocytes. The implantation rate (the number of observed gestational sacs divided by the number of embryos transferred for each patient), clinical pregnancy (the presence of a gestational sac with fetal heart beat on vaginal ultrasound), early miscarriage (spontaneous loss of a clinical pregnancy $\leq 12$ weeks of gestation) and ongoing pregnancy (pregnancies continued more than 12 weeks after ET) rates were considered as secondary outcomes.

\section{Statistical Analysis}


The study sample size (85 subjects in each group) was estimated using Epi-Info software with considering a difference of 0.5 , significance level (alpha level) of 0.05 and $80 \%$ power. Statistical analysis was done using the Statistical Package for Social Sciences (SPSS Inc., Chicago, IL, USA) version 23.0. The comparisons of continuous variables between groups were provided by student's t-test and presented as mean \pm standard deviation (SD). The chi-square test was used for comparing the categorical variables between groups. The statistical significant level was considered at $p$ value $<0.05$.

\section{Results}

The study subjects' sampling flow chart according to the Consolidated Standards of Reporting Trials (CONSORT) guideline was presented in Figure 1. During the study period, 150 infertile patients were evaluated for participation in the study. Of these, 34 women were excluded due to non-eligibility for entering the study and a total of 116 women were randomly assigned to early start antagonist group $(n=58)$ and conventional antagonist group $(n=58)$. The baseline characteristics of patients were shown in Table 1 and according to the results, the two groups were comparable. There was no significant difference in terms of the women's age and body mass index, cause and duration of infertility, total antral follicle count and baseline hormones assay between groups.

The comparison of the $\mathrm{COH}$ cycle and pregnancy outcomes between groups were presented in Table 2 . The analysis showed that the two groups did not have a statistically significant difference in terms of the ovarian stimulation duration and the total dose of used gonadotropins. Although, the total number of MII oocytes in early start of antagonist group was significantly higher than that of in control group ( $P=0.04)$. The rates of no oocyte and no embryo development were similar between groups. Also, the all-freeze rate in early start of antagonist group was higher than that of in the control group, however the difference was not statistically significant $(P=0.08)$. There was no significant difference in the number of transferred embryos and endometrial thickness at ET day between groups. In the following, clinical and ongoing pregnancy rates per ET in early start of antagonist group were significantly higher than those of in control group $(P=0.02$ and $P=0.03$, respectively); however, the implantation and miscarriage rates were similar between groups (Table 2). 
Table 1

The patients' characteristics in the study groups

\begin{tabular}{|c|c|c|c|}
\hline Variables & $\begin{array}{l}\text { Early start antagonist } \\
\text { protocol } \\
\qquad(n=58)\end{array}$ & $\begin{array}{l}\text { Flexible antagonist } \\
\text { protocol } \\
(n=58)\end{array}$ & $\begin{array}{l}\mathrm{P}- \\
\text { value }\end{array}$ \\
\hline Age of women (years) & $38.1 \pm 4.64$ & $38.9 \pm 4.0$ & 0.28 \\
\hline Women's BMI $\left(\mathrm{kg} / \mathrm{m}^{2}\right)$ & $27.50 \pm 0.46$ & $26.80 \pm 0.48$ & 0.55 \\
\hline $\begin{array}{l}\text { Duration of infertility } \\
\text { (years) }\end{array}$ & $5.02 \pm 5.01$ & $6.50 \pm 5.41$ & 0.13 \\
\hline \multicolumn{4}{|l|}{ Causes of infertility $n(\%)$} \\
\hline Ovarian factor & $28(48.3)$ & $31(53.3)$ & 0.71 \\
\hline Ovarian factor\& Male & $30(51.7)$ & $27(46.6)$ & \\
\hline Total antral follicles count & $4.75 \pm 1.87$ & $4.70 \pm 2.28$ & 0.89 \\
\hline Basal serum FSH (mIU/m) & $8.2 \pm 3.0$ & $8.5 \pm 2.7$ & 0.51 \\
\hline Basal serum LH (mlU/ml) & $4.41 \pm 4.91$ & $5.53 \pm 7.03$ & 0.24 \\
\hline Serum AMH (ng/ml) & $1.0 \pm 0.9$ & $0.8 \pm 0.6$ & 0.70 \\
\hline Basal Estradiol (pg/ml) & $44.89 \pm 4.42$ & $52.37 \pm 4.83$ & 0.14 \\
\hline $\begin{array}{l}\text { Basal progesterone } \\
(\mathrm{ng} / \mathrm{ml})\end{array}$ & $0.48 \pm 0.03$ & $0.44 \pm 0.03$ & 0.85 \\
\hline No. of previous IVF & $0.43 \pm 0.85$ & $0.36 \pm 0.64$ & 0.90 \\
\hline
\end{tabular}

Descriptive data were presented as Mean \pm SD. P-value $\leq 0.05$ was considered statistically significant. BMI: body mass index; No.; number, FSH; follicle stimulating hormone; $\mathrm{LH}$; luteinizing hormone; $\mathrm{AMH}$; anti-Müllerian hormone, IVF; in-vitro fertilization 
Table 2

Comparison of the cycle and pregnancy outcomes between groups

\begin{tabular}{|c|c|c|c|}
\hline & $\begin{array}{l}\text { Early start antagonist } \\
\text { protocol } \\
\qquad(n=58)\end{array}$ & $\begin{array}{l}\text { Flexible antagonist } \\
\text { protocol } \\
(n=58)\end{array}$ & P-value \\
\hline Duration of stimulation (days) & $10.12 \pm 2.28$ & $10.0 \pm 1.50$ & 0.76 \\
\hline No. of used antagonist ampoule & $10.12 \pm 2.28$ & $3.93 \pm 1.32$ & $<0.0001$ \\
\hline $\begin{array}{l}\text { Total ampoules of used } \\
\text { gonadotropins (75IU) }\end{array}$ & $47.2 \pm 12.7$ & $43.7 \pm 10.4$ & 0.11 \\
\hline Serum estradiol on HCG day & $524 \pm 78.29$ & $358 \pm 51.21$ & 0.23 \\
\hline Serum progesterone on HCG day & $0.61 \pm 0.37$ & $0.52 \pm 0.28$ & 0.17 \\
\hline Serum LH level on HCG day & $2.60 \pm 3.14$ & $3.23 \pm 5.66$ & 0.52 \\
\hline No. of retrieved oocytes & $4.17 \pm 2.89$ & $3.20 \pm 2.47$ & 0.07 \\
\hline No. of metaphase II oocytes & $3.35 \pm 2.55$ & $2.46 \pm 2.20$ & 0.04 \\
\hline Fertilization rate & $0.77 \pm 0.24$ & $0.79 \pm 0.25$ & 0.5 \\
\hline embryo $\quad$; (\%) No. of obtained & $2.61 \pm 1.45$ & $2.13 \pm 1.47$ & 0.17 \\
\hline No. of top quality embryos; $n(\%)$ & $1.68 \pm 1.63$ & $1.37 \pm 1.41$ & 0.30 \\
\hline Cycle cancellation rate; $\mathrm{n}(\%)$ & $5(8.6)$ & $2(3.4)$ & 0.43 \\
\hline No oocyte rate; $\mathrm{n}(\%)$ & $2(3.4)$ & $3(5.2)$ & 0.54 \\
\hline No embryo rate; $n$ (\%) & $15(25.8)$ & $10(17.2)$ & 0.16 \\
\hline All-freeze rate; $\mathrm{n}(\%)$ & $21(36.2)$ & $14(24.1)$ & 0.08 \\
\hline No. of embryos transferred & $2.20 \pm 0.67$ & $1.73 \pm 1.08$ & 0.13 \\
\hline $\begin{array}{l}\text { Endometrial thickness at ET day } \\
(\mathrm{mm})\end{array}$ & $9.34 \pm 1.14$ & $9.08 \pm 0.98$ & 0.19 \\
\hline Implantation rate & $0.58 \pm 0.39$ & $0.33 \pm 0.28$ & 0.36 \\
\hline Clinical pregnancy rate per ET (\%) & $6 / 15(40)$ & $3 / 29(11.1)$ & 0.02 \\
\hline Miscarriage rate per ET (\%) & $1 / 15(6.6)$ & $1 / 29(3.7)$ & 0.53 \\
\hline Ongoing pregnancy rate per ET (\%) & $5 / 15(33.3)$ & $2 / 29(6.9)$ & 0.03 \\
\hline
\end{tabular}


HCG: human chorionic gonadotropin; LH: luteinizing hormone; ET: embryo transfer; Descriptive data were presented as Mean \pm SD. P-value $\leq 0.05$ was considered statistically significant.

\section{Discussion}

In the present study, the outcomes of $\mathrm{COH}$ cycle and pregnancy after early onset $\mathrm{GnRH}$-ant protocol were compared with conventional $\mathrm{GnRH}$-ant protocol in patients with POR diagnosis. Our results revealed higher number of retrieved and MII oocytes and also higher rate of clinical pregnancy following fresh embryo transfer in early start GnRH-ant protocol than the conventional group, although the rates of implantation and ongoing pregnancy were not significantly different between groups. It is worth noting that this strategy did not affect the total dose of gonadotropins, duration of stimulation and cycle cancellation rate in these patients.

In this regards, Kolibianakis et al. in a randomized clinical study concluded that high exposure of the genital tract to $\mathrm{LH}$ and $\mathrm{E}_{2}$ in the early follicular phase is associated with a reduced chance of pregnancy in cycles stimulated with recombinant FSH and GnRH-ant for IVF/ICSI, therefore, it is assumed that the endocrine environment of the early follicular phase in antagonist cycles might be related to the reproductive outcome (9). In agreement with them, in our study higher MII oocytes and clinical pregnancy rate after fresh embryo transfer were observed in early onset $\mathrm{GnRH}$-ant protocol.

The beneficial effect of early follicular phase $\mathrm{GnRH}$-ant on improving the number of retrieved and MII oocytes in normal responder patients were shown in previous studies; however, they have reported no significant positive effect on pregnancy rate in these patients $(14,15)$. Park et al. in a retrospective study of normal responder women, concluded that the modified early antagonist start protocol may improve the mature oocyte yield, possibly via enhanced follicular synchronization, while resulting in superior CPR as compared to the conventional antagonist protocol, which requires to be studied further in prospective randomized controlled trials. Regarding to patients with POR diagnosis, the most of previous studies with early onset $\mathrm{GnRH}$-ant protocol, estradiol pre-treatment ( $\mathrm{E}_{2}$ priming) was used in late luteal phase prior to the start of the antagonist protocol $(7,8,16)$; hence, the results of the present study were not comparable to them. In whatever way regardless of how, most of the mentioned studies have shown evidence of improved ovarian stimulation outcomes $(7,8,16,17)$ and pregnancy rates $(8)$ after early onset $\mathrm{GnRH}$-ant protocol with $\mathrm{E}_{2}$ priming in POR patients.

In the flexible type, the early onset of the antagonist appears to have beneficial effects on the late onset of the antagonist. On the other hand, due to the possibility of LH surge occurring earlier than the sixth day, stimulation and release of oocytes before puncture, especially in POR individuals with fewer eggs, early onset of antagonist will prevent the loss of these limited growing follicles (18). Furthermore, early onset of $\mathrm{GnRH}$ antagonist may lead to better follicular synchronization, which increases follicle maturation. It is difficult to determine the exact time of increasing FSH level at interphase due to intrinsic changes in FSH because it can occur before or at the beginning of the follicular phase; therefore it is believed that the interphase peak of FSH can be suppressed using early GnRH antagonist onset at the 
beginning of the menstrual cycle and primary FSH suppression may be helpful in achieving follicles coordination (19).

Nevertheless, the present study has some limitations and some strength points that should be mentioned. The strength of present study was the randomized clinical trial methodology and selection of homogenous population of POR patients. The rate of all-freeze case in experimental group was a little more and the results of frozen embryo transfer had not been determined until the time of manuscript writing; therefore, the power of present study for comparing the clinical and cumulative pregnancy rates between groups is limited, although these issues were our secondary outcomes and we suggested further studies with larger sample size to evaluate pregnancy outcome as main objective.

\section{Conclusion}

In conclusion, the early start GnRH-ant protocol can improve the number of retrieved and MII oocytes and pregnancy outcome after fresh embryo transfer; however, larger randomized studies are required to compare the pregnancy outcomes after this protocol versus other $\mathrm{COH}$ protocols with considering costeffectiveness issue.

\section{Abbreviations}

ART: assisted reproduction technology; AFC: antral follicle count; $\mathrm{AMH}$ : anti-Müllerian hormone; $\mathrm{COH}$ : controlled ovarian response; $\mathrm{E}_{2}$ : estradiol; $\mathrm{GnRH}$-ant: gonadotropin-releasing hormone antagonist; $\mathrm{HMG}$ : human menopausal gonadotrophins; hCG: human chorionic gonadotropin; SD: standard deviation; LH: luteinising hormone; IVF/ICSI: in -vitro fertilization/ intra-cytoplasmic sperm injection; POR: poor ovarian response;

\section{Declarations}

\section{Ethics approval and consent to participate}

The Institutional Review Boards and the Ethics Committees of Tehran University of Medical Sciences approved study trial (ethics code: IR.TUMS.MEDICINE.REC.1398.544). All procedures conducted in the present study involving human participants were in accordance with the ethical standards of Tehran University of Medical Sciences and the 1964 Helsinki declaration and its later amendments or comparable ethical standards. The eligible patients signed written informed consent prior to participation in this clinical trial.

\section{Consent for publication}

Not applicable. 


\section{Availability of data and materials}

The datasets used and/or analyzed during the current study are available from the corresponding authors on reasonable request.

\section{Funding}

This study did not receive any specific grant from funding agencies in the public, commercial, or not-forprofit sectors.

\section{Competing interests}

All authors have nothing to disclose.

\section{Authors' contributions}

TE, LK and AM designed the research. TE, AM, LK, MFM and SHM contributed in patient's selection, data collection, interpretation of data and manuscript writing/editing. TE and LK wrote the manuscript. AA helped in the analysis of the data. All authors read and approved the final manuscript.

\section{Acknowledgments}

We would like to thank all the colleagues in Arash women's Hospital and all of the participants for their assistance in this study.

\section{References}

1. Albano C, Felberbaum R, Smitz J, et al. Ovarian stimulation with HMG: results of a prospective randomized phase III European study comparing the luteinizing hormone-releasing hormone (LHRH)antagonist cetrorelix and the LHRH-agonist buserelin. Human reproduction. 2000;15(3):526-31.

2. Abdul-Hameed KM, Al-Kawaz UM, Al-Hilli NM. Early and Short Follicular GnRH Antagonist (Sandwich) Protocol Versus Conventional GnRH Antagonist Protocol in Normal Responders. Iraqi Journal of Embryos and Infertility Researches. 2019;9 (1):68-88.

3. Shapiro DB, Mitchell-Leef D, Carter M, Nagy ZP. Ganirelix acetate use in normal-and poor-prognosis patients and the impact of estradiol patterns. Fertility and sterility. 2005;83(3):666-70.

4. Al-Jeborry MM. Comparison of sandwich, conventional antagonist and microdose protocols in poor responders. 2019. 
5. Inal ZO, Yilmaz N, Inal HA, Hancerliogullari N, Coskun B. Are there any differences between antagonist administration on days $<6$ and 6 of $\mathrm{COH}$ on assisted reproductive technique outcomes? Journal of the Chinese Medical Association. 2017;20:1e5.

6. Kolibianakis EM, Albano C, Camus M, Tournaye H, Van Steirteghem AC, Devroey P. Initiation of gonadotropin-releasing hormone antagonist on day 1 as compared to day 6 of stimulation: effect on hormonal levels and follicular development in in vitro fertilization cycles. The Journal of Clinical Endocrinology \& Metabolism. 2003;88(12):5632-7.

7. Cakmak H, Tran ND, Zamah AM, Cedars MI, Rosen MP. A novel "delayed start" protocol with gonadotropin-releasing hormone antagonist improves outcomes in poor responders. Fertility and sterility. 2014;101(5):1308-14.

8. Maged AM, Nada AM, Abohamila F, Hashem AT, Mostafa WA, Elzayat AR. Delayed start versus conventional $\mathrm{GnRH}$ antagonist protocol in poor responders pretreated with estradiol in luteal phase: a randomized controlled trial. Reproductive Sciences. 2015;22(12):1627-31.

9. Kolibianakis EM, Albano C, Kahn J, et al. Exposure to high levels of luteinizing hormone and estradiol in the early follicular phase of gonadotropin-releasing hormone antagonist cycles is associated with a reduced chance of pregnancy. Fertility and sterility. 2003;79(4):873-80.

10. Tannus S, Weissman A, Boaz M, et al. The effect of delayed initiation of gonadotropin-releasing hormone antagonist in a flexible protocol on in vitro fertilization outcome. Fertility and sterility. 2013;99(3):725-30.

11. Hamdine O, Macklon N, Eijkemans M, et al. Comparison of early versus late initiation of GnRH antagonist co-treatment for controlled ovarian stimulation in IVF: a randomized controlled trial. Human reproduction. 2013;28(12):3227-35.

12. Ferraretti AP, La Marca A, Fauser BC, Tarlatzis B, Nargund G, Gianaroli L. ESHRE consensus on the definition of 'poor response' to ovarian stimulation for in vitro fertilization: the Bologna criteria. Hum Reprod. 2011;26(7):1616-24. Epub 2011/04/21.

13. Cummins J, Breen T, Harrison K, Shaw J, Wilson L, Hennessey J. A formula for scoring human embryo growth rates in in vitro fertilization: its value in predicting pregnancy and in comparison with visual estimates of embryo quality. Journal of In Vitro Fertilization and Embryo Transfer. 1986;3(5):284-95.

14. Younis JS, Soltsman S, Izhaki I, Radin O, Bar-Ami S, Ben-Ami M. Early and short follicular gonadotropin-releasing hormone antagonist supplementation improves the meiotic status and competence of retrieved oocytes in in vitro fertilization-embryo transfer cycles. Fertility and sterility. 2010;94(4):1350-5.

15. Blockeel C, Riva A, De Vos M, Haentjens P, Devroey P. Administration of a gonadotropin-releasing hormone antagonist during the 3 days before the initiation of the in vitro fertilization/intracytoplasmic sperm injection treatment cycle: impact on ovarian stimulation. A pilot study. Fertility and sterility. 2011;95(5):1714-9. e2. 
16. Davar R, Neghab N, Naghshineh E. Pregnancy outcome in delayed start antagonist versus microdose flare GnRH agonist protocol in poor responders undergoing IVF/ICSI: An RCT. International Journal of Reproductive BioMedicine. 2018;16(4):255.

17. Ashrafi M, Arabipoor A, Yahyaei A, Zolfaghari Z, Ghaffari F. Does the "delayed start" protocol with gonadotropin-releasing hormone antagonist improve the pregnancy outcome in Bologna poor responders? a randomized clinical trial. Reproductive Biology and Endocrinology. 2018;16(1):1-7.

18. Hamdine O, Broekmans FJ, Eijkemans MJC, Lambalk CB, Fauser BM, Laven JSE, NS Macklon, CETRO trial study group .Early initiation of gonadotropin-releasing hormone antagonist treatment results in a more stable endocrine milieu during the mid-and late-follicular phases: a randomized controlled trial comparing gonadotropin-releasing hormone antagonist initiation on cycle day 2 or 6 . Fertility and sterility, 2013. 100(3): p. 867-874.

19. ParkCW, HwangYl, KooHS, KangIS, YangKM, SonglO. Early gonadotropin-releasing hormone antagonist start improves follicular synchronization and pregnancy outcome as compared to theconventional antagonist protocol. Clin Exp Reprod Med, 2014 Dec;41(4):158-64.

\section{Figures}




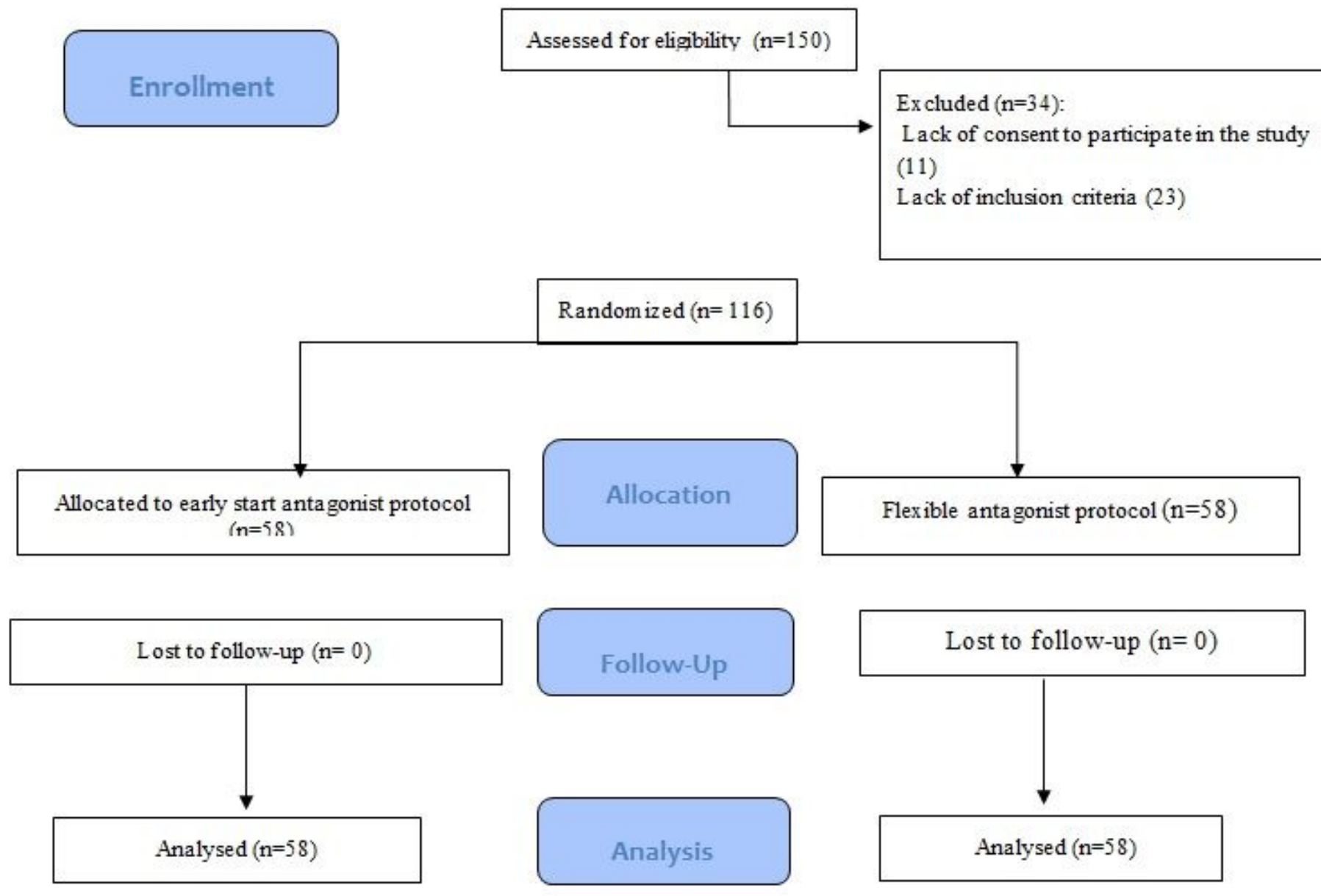

Figure 1

The study subjects' sampling flow chart 